\title{
Effect of Stirring on Chemically Deposited ZnO Thin Films
}

\author{
M. ÖNAL* AND B. AltiokKA \\ Bilecik Şeyh Edebali University, Bilecik 11210, Turkey \\ (Received January 29, 2020; in final form March 20, 2020)
}

\begin{abstract}
In this study, the effects of stirring rate were investigated while $\mathrm{ZnO}$ thin films were produced by the chemical bath deposition method. Five different samples were produced by mixing the prepared solution at $0,300,600$, 900, and $1200 \mathrm{rpm}$ cycles. Scanning electron microscope showed that $\mathrm{ZnO}$ nanorods were formed very rarely when the solution was not stirred and that the nanorods became more frequent as the stirring rate increased. X-ray diffraction results showed that all films formed hexagonal wurtzite structure and peak intensities were compatible with the ASTM card. According to the absorbance measurements, the energy band gaps of the samples were between $3.02 \mathrm{eV}$ and $3.97 \mathrm{eV}$. When the visualization of the obtained films was examined, it was observed that as the stirring rate increased, $\mathrm{ZnO}$ adhered well on the glass substrates. It was observed that the film adhering on the glass substrate at $0 \mathrm{rpm}$ was very weak, whereas the film adhered very well on the glass surface as the solution stirring rate increased.
\end{abstract}

DOI: 10.12693/APhysPolA.137.1209

PACS/topics: ZnO, chemical bath deposition, stirring rate, thin film

\section{Introduction}

Nanocrystalline semiconductor materials are of great interest due to their size-dependent properties and wide application areas [1]. In particular, $\mathrm{ZnO}$ is a semiconductor material that attracts the attention of researchers with its $n$-type conductivity, $3.3 \mathrm{eV}$ energy band gap, and potential applications. The potential uses of $\mathrm{ZnO}$ are piezoelectrical devices, chemical sensors, electroluminescent devices, solid-state emission, solar cells, transparent electrodes, photo-catalysts, and ultraviolet laser diodes [2-4]. The main reason for this interest is that zinc oxide $(\mathrm{ZnO})$ is a versatile semiconductor with multifunctional properties that can be easily synthesized in various structural forms [5]. The development of highquality $\mathrm{ZnO}$ thin films has been made possible by advances in deposition techniques. Some of these techniques are ultrasonic spray pyrolysis, chemical bath deposition, sol-gel, RF magnetron spray, precipitation in water solution, hydrothermal synthesis, pulse laser deposition [6-8]. Among all these methods, chemical bath deposition (CBD) is a highly preferred method because of its low cost, high efficiency, simplicity and compliance with large scale production [9].

In the literature, the effects of the stirring rate on the growth of CdS films produced by the chemical bath deposition method have already been investigated. In this mentioned experiment, $\mathrm{CdS}$ was obtained by mixing at 250, 350, $450 \mathrm{rpm}, \mathrm{X}$-ray diffraction (XRD) peak densities increased and film thicknesses were 40, 80, and $120 \mathrm{~nm}$, respectively [10]. In addition, Zhang et al. investigated various properties of $\mathrm{ZnS}$ films deposited at different stirring rates $(150-525 \mathrm{rpm})$ by the CBD method.

*corresponding author; e-mail: metehan.onal@bilecik.edu.tr
It was seen that when the stirring rates increased, the films' thickness also increased [11]. However, the effects of stirring on $\mathrm{ZnO}$ thin films have not been investigated in the literature.

In this study, the effects of stirring rate when producing $\mathrm{ZnO}$ thin films with chemical bath deposition were investigated for the first time. The stirring rate has significant effects on structural and surface morphology. These effects were investigated in detail.

The visual photos of the thin films reveal the effect of the stirring rate on the surface of samples. As can be seen from these photos, the film thickness increased with an increasing stirring rate. UV measurements showed that when the solution was not stirred, the absorbance values of the films were relatively low such as 0.45 and when stirred, absorbance value increased to 2.00 above the wavelength of $400 \mathrm{~nm}$. It was observed that the energy bandgap of the films decreased from $3.97 \mathrm{eV}$ to $3.02 \mathrm{eV}$.

\section{Experimental details}

In this study, the chemical bath deposition method was used to produce $\mathrm{ZnO}$ thin films. Before starting the deposition process, the bath container was washed with $10 \% \mathrm{HCl}$ acid and the glass substrate was washed with acetone and rinsed with deionized water. After then, $100 \mathrm{ml}$ deionized water was placed in the bath container and heating was started. In the bath container, when the deionized water temperature reached $30^{\circ} \mathrm{C}, 65 \mathrm{mM}$ $\mathrm{ZnCl}_{2}$ and $6 \mathrm{mM}$ EDTA were dissolved. The $\mathrm{pH}$ of the solution was adjusted to 10.25 using $\mathrm{NaOH}$. While the first sample was being produced, the solution was heated to the $85^{\circ} \mathrm{C}$ and the deposition was completed in $25 \mathrm{~min}$. Besides, in this experiment, the solution was not stirred. This sample was named as rpm0. 


\section{TABLE I}

The summarized details for $\mathrm{ZnO}$ experiment, where $P h=10.25$, temperature $T=85 \pm 2{ }^{\circ} \mathrm{C}$.

\begin{tabular}{l|c|c|c}
\hline \hline \multicolumn{1}{c|}{ Exp. } & $\mathrm{ZnCl}_{2}[\mathrm{mM}]$ & EDTA $[\mathrm{mM}]$ & $\begin{array}{c}\text { Stirring rate } \\
{[\mathrm{rev} / \mathrm{min}]}\end{array}$ \\
\hline rpm0 & 65 & 6 & 0 \\
rpm300 & 65 & 6 & 300 \\
rpm600 & 65 & 6 & 600 \\
rpm900 & 65 & 6 & 900 \\
rpm1200 & 65 & 6 & 1200
\end{tabular}

Under the same conditions mentioned above, four other different samples were produced being stirred at $300,600,900$, and $1200 \mathrm{rpm}$ and the samples were named as rpm300, rpm600, rpm900, and rpm1200. Each sample was rinsed using pressurized water and allowed to dry at room temperature. All production conditions of the samples were given in Table I.

Optical properties of $\mathrm{ZnO}$ thin film samples were studied with JASCO V-530 with double beam UV-vis spectrometer. The morphological characteristic of the films was observed using a Zeiss SUPRA 40VP SEM. The crystal structure was examined using a PANalytical Empyrean XRD. For analysis of the Fourier transform infrared photometer (FTIR) a PERKIN Elmer device was used.

\section{Results and discussion}

\subsection{Structural analysis of $\mathrm{ZnO}$ films}

The thicknesses of the obtained samples were calculated by the gravimetric method. The thickness of the film obtained without stirring was calculated at an average of $175 \mathrm{~nm}$ and the other samples were calculated to be $\approx 650 \mathrm{~nm}$. Since the metal ions in the solution are heavier than water molecules, when the solution was not mixed they precipitated to the bottom and did not participate in the chemical reaction. When the solution was stirred, since metal ions are heavier than water molecules, they were exposed to more centrifugal forces. Metal ions that were subjected to more centrifugal forces were directed to the glass surface, causing more reactions to occur on the glass substrate and increasing the film thicknesses.

The structural properties of $\mathrm{ZnO}$ thin films were investigated by using XRD. The XRD patterns are given in Fig. 1. As seen in Fig. 1, all films had a hexagonal structure. To calculate the preferred orientations of the films we used the following formula:

$$
T . C .=\frac{I(h k l)}{I_{0}(h k l)} /\left[\frac{1}{N} \sum_{N} \frac{I(h k l)}{I_{0}(h k l)}\right],
$$

where $I_{0}(h k l)$ is the standard density of the $(h k l)$ plane given on the ASTM card, and $I(h k l)$ is the measured relative density of the $(h k l)$ plane. Calculated texture coefficient values are given in Table II. There one can see that the preferred orientation has shifted from plane (010) to plane (002).



Fig. 1. XRD diffraction data of $\mathrm{ZnO}$ thin films obtained at different stirring rates.

\section{TABLE II}

Calculated texture coefficients at preferred orientation of $\mathrm{ZnO}$ films.

\begin{tabular}{c|c|c|c|c|c}
\hline \hline Exp. & rpm0 & rpm300 & rpm600 & rpm900 & rpm1200 \\
\hline T.C.(010) & 1.298 & 0.617 & 1.147 & 0.888 & 0.376 \\
T.C.(002) & 0.919 & 1.479 & 1.048 & 1.143 & 1.330 \\
T.C.(011) & 0.782 & 0.902 & 0.803 & 0.968 & 0.991
\end{tabular}

The crystallite sizes (cs $[\mathrm{nm}])$ and average crystallite sizes are given in Table III. They were calculated using the Debye-Scherrer equation

$$
c s=\frac{0.089 \times 180 \lambda}{314 \beta \cos \left(\theta_{C}\right)}
$$

where $\lambda$ is the wavelength of X-ray radiation (1.54056 $\AA$ ), $\beta$ is the full width half maximum (FWHM), and $2 \theta_{C}$ is the peak center [12]. It is seen in Table III that the average crystallite size of the film obtained without stirring was $16 \mathrm{~nm}$. On the other hand, the average crystallite sizes of the other films were $22 \mathrm{~nm}$.

\subsection{Optical properties of the $\mathrm{ZnO}$ films}

The absorbance versus wavelength was recorded by using a UV-vis spectrophotometer and absorbance plots are given in Fig. 2. 
The crystallite sizes $c s[\mathrm{~nm}]$ and energy band gaps of the $\mathrm{ZnO}$ films.

TABLE III

\begin{tabular}{l|c|c|c|c|c|c}
\hline \hline Exp. & $\operatorname{cs}(010)[\mathrm{nm}]$ & $\operatorname{cs}(002)[\mathrm{nm}]$ & $\operatorname{cs}(011)[\mathrm{nm}]$ & Average cs & Band gap [eV] & Thickness [nm] \\
\hline rpm0 & 15 & 15 & 19 & 16 & 3.97 & 175 \\
rpm300 & 19 & 15 & 29 & 21 & 3.02 & 650 \\
rpm600 & 19 & 29 & 19 & 22 & 3.12 & 650 \\
rpm900 & 19 & 29 & 19 & 22 & 3.14 & 650 \\
rpm1200 & 19 & 29 & 29 & 22 & 3.10 & 650
\end{tabular}

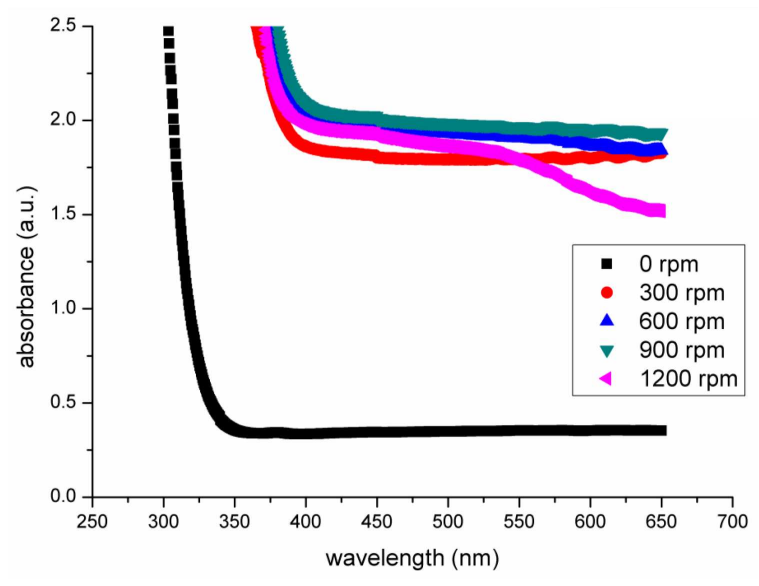

Fig. 2. Absorbance measurements at wavelengths between 300 and $650 \mathrm{~nm}$.

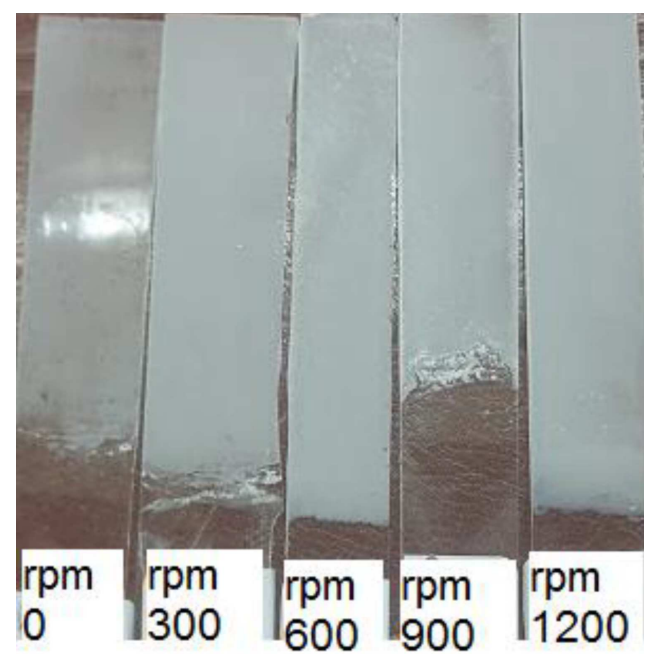

Fig. 3. The photographs of the $\mathrm{ZnO}$ films.

When the film thickness increased, absorbance values increased, and transparency decreased. As seen in Fig. 3, while the other films were not more transparent, that of the film obtained without stirring was quite transparent and the nanoparticles did not adhere well to the glass surface. When the other samples produced by mixing the solution were examined, it was seen that the films covered the surface quite well and formed a homogeneous and compact structure. In particular, the sample obtained at $1200 \mathrm{rpm}$ appeared with covered densely by nanorods.

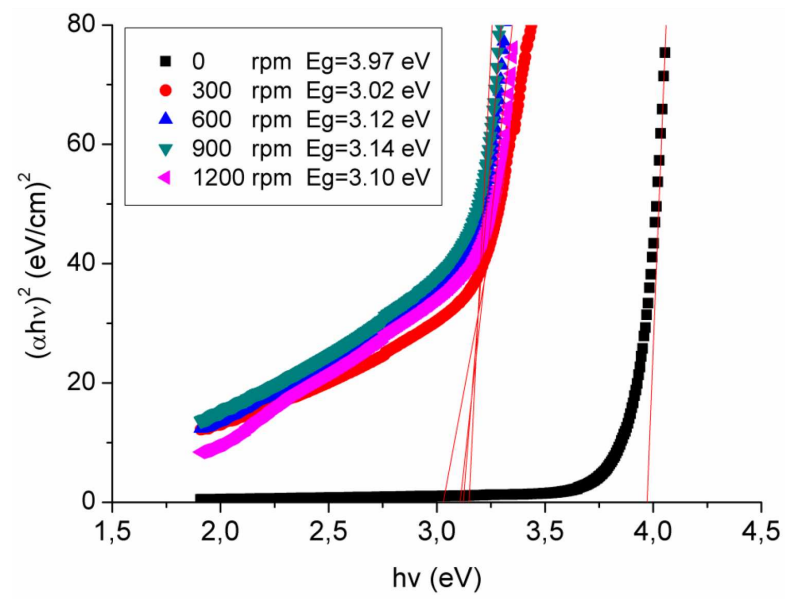

Fig. 4. The Tauc plots and energy band gaps for $\mathrm{ZnO}$ films.

In case of Fig. 2, the absorbance of the film obtained without stirring the solution (rpm0) was seen to be about 5 times lower than that of the other films. When rpm0 and others were compared, the sharp increasing absorbance value was quite different. The sharp increasing value of the film obtained in rpm0 was about $325 \mathrm{~nm}$ while the others were about $375 \mathrm{~nm}$. The band gaps were strongly affected by the sharp increase of absorbance values.

The Tauc relation used to calculate the optical energy bandgap of thin films is given by

$$
(\alpha h \nu)^{2}=A\left(h \nu-E_{g}\right)^{n} .
$$

In the above equation $\alpha$ is the absorption coefficient, $h \nu$ is the photon energy, $E_{g}$ is the optical band gap of the sample, $A$ is a constant, while $n=1 / 2$ for directly allowed transitions [13].

Figure 4 shows that the energy band gap of the film obtained at $\mathrm{rpm} 0$ was $3.97 \mathrm{eV}$, while the average band gap for the other films was $3.10 \mathrm{eV}$. When the literature is examined, it is seen that the band gap of $\mathrm{ZnO}$ thin films can have different values such as 3.2, 3.3, 3.37, $3.40 \mathrm{eV}$ [14-17]. The reduction of the band gap indicates that the crystallite size was increased and the film thickness was also increased, which can be seen in Table III.

\subsection{FTIR of the ZnO films}

The Fourier transform infrared spectroscopy (FTIR) photometer was used to investigate the vibration properties of synthesized materials [18]. In order to determine 


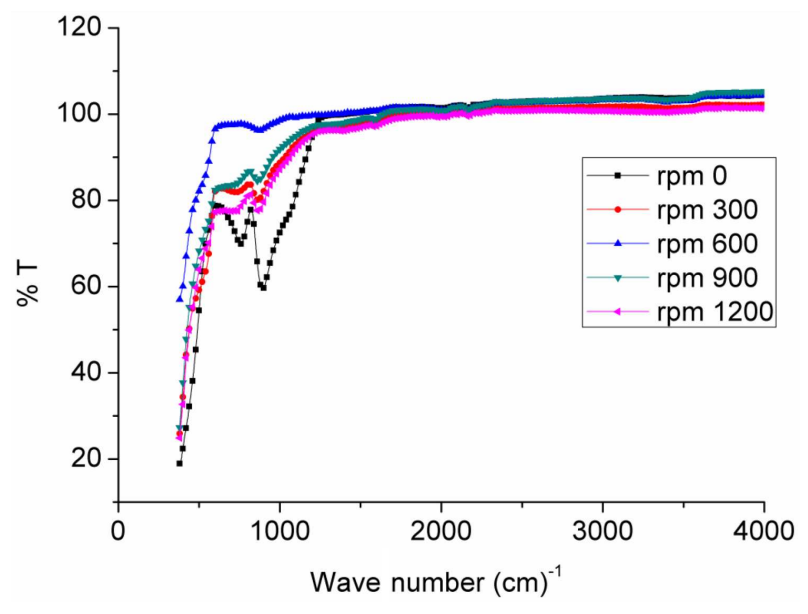

Fig. 5. FTIR spectra vs. wave number for $\mathrm{ZnO}$ films.

TABLE IV

Average width and average length of samples.

\begin{tabular}{l|c|c|c}
\hline \hline \multicolumn{1}{c|}{ Exp. } & $\begin{array}{c}\text { Average } \\
\text { width [nm] }\end{array}$ & $\begin{array}{c}\text { Average } \\
\text { length [nm] }\end{array}$ & $\begin{array}{c}\text { Stirring } \\
\text { rate [rev/min] }\end{array}$ \\
\hline rpm0 & 580 & 3750 & 0 \\
rpm300 & 660 & 3150 & 300 \\
rpm600 & 420 & 2500 & 600 \\
rpm900 & 330 & 1650 & 900 \\
rpm1200 & 250 & 1300 & 1200
\end{tabular}

the structure and purity of metal nanoparticles, infrared beams were used. Metal oxides generally give absorption bands in the fingerprint region, i.e., below $1000 \mathrm{~cm}^{-1}$ arising from inter-atomic vibrations [19]. FTIR measurements were performed in the range of 4000 to $300 \mathrm{~cm}^{-1}$ wave counts. Figure 5 shows the FTIR spectra of $\mathrm{ZnO}$ nanoparticles. $\mathrm{ZnO}$ absorption stretching was observed at $\approx 900 \mathrm{~cm}^{-1}$ and the band at $600 \mathrm{~cm}^{-1}$ was the stretching mode of $\mathrm{ZnO}$. Peaks observed around $593 \mathrm{~cm}^{-1}$ and around $925 \mathrm{~cm}^{-1}$ corresponded to the $\mathrm{Zn}-\mathrm{O}$ vibrations. This confirmed the wurtzite structure formation of the film [20].

\subsection{SEM analysis of the $\mathrm{ZnO}$ films}

The surface morphology of $\mathrm{ZnO}$ thin films produced by the chemical bath deposition method was examined using scanning electroscopy (SEM). The 10000 times magnified SEM images of five different samples produced by stirring at different rates are given in Fig. 6.

It is seen from these images that the stirring rate has a significant effect on the surface properties of the films. When the stirring rate was zero, the nanorods were very rare and their length $3750 \mathrm{~nm}$ and diameter $580 \mathrm{~nm}$. Figure 6 shows that as the stirring rate of the solutions increases, the nanorods become more frequent and their width and length decrease. The average width of these nanorods decreased to $250 \mathrm{~nm}$ at $1200 \mathrm{rpm}$. The smaller

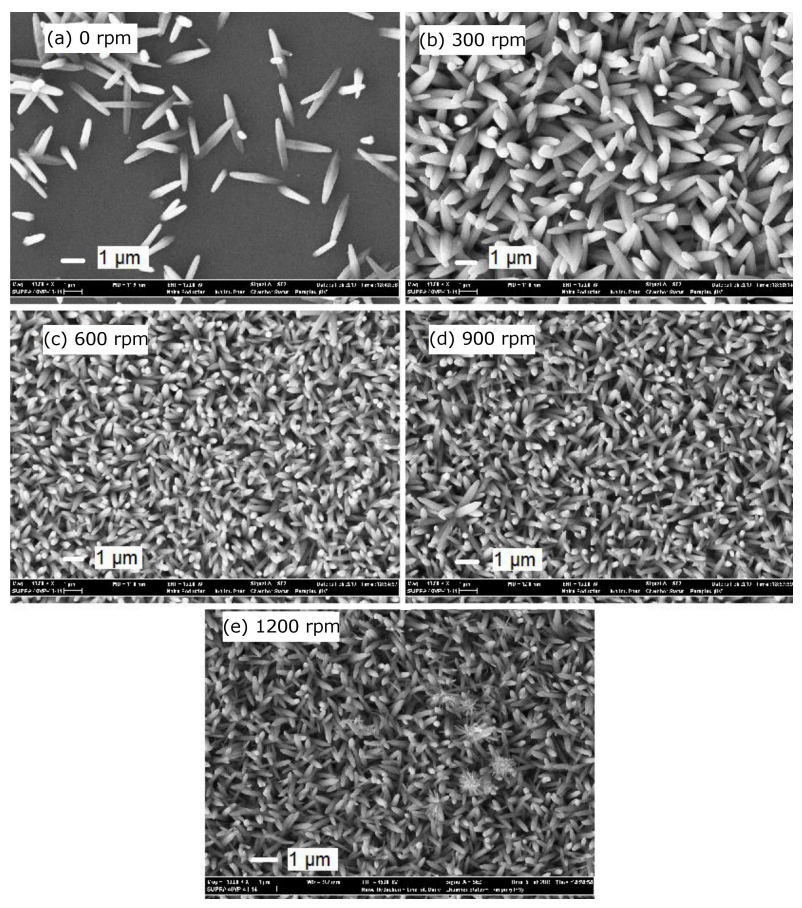

Fig. 6. SEM images of $\mathrm{ZnO}$ thin films magnified 10,000 times (a) $0 \mathrm{rpm}$, (b) $300 \mathrm{rpm}$, (c) $600 \mathrm{rpm}$, (d) $900 \mathrm{rpm}$, and (e) $1200 \mathrm{rpm}$.

the nanorod dimensions, the higher the surface area and the roughness, which can make the resulting thin films a suitable material for gas sensors.

The average nanorod sizes varying with stirring rates are given in Table IV. The images of SEM also show that the films obtained with stirring were dense.

\section{Conclusions}

In this study, the effects of the stirring rate on $\mathrm{ZnO}$ thin films produced by the chemical bath deposition method were investigated for the first time. When the solution was not stirred, the film had a low thickness such as $175 \mathrm{~nm}$ although the average of the other samples $650 \mathrm{~nm}$. The structural properties were realized by using the XRD pattern. The sample obtained without stirring the solution had a low XRD peak intensity. This may be because the film was thin or the crystallization was not good. The band gaps of the films were estimated by using absorbance measurements. These plots showed a significant result. When the solution was not mixed, it was observed that the band gaps of the films were decreased up to $0.95 \mathrm{eV}$. The surface analysis was examined by SEM images. SEM images revealed that the size of nanorods was decreased as the stirring rate was increased. The photos of the surface of the obtained samples were taken. As the solution stirring rate increased, it was observed that the surfaces of the obtained samples were more densely coated and their transparency decreased. It is a known fact that the metal ions are heavier than 
water molecules. Thus, when the solution was mixed, by the effect of the centrifugal force, the metal ions were thrown into the inner wall of the bath container and the surface of the glass substrate.

\section{References}

[1] J. Kathalingam, A. Ambika, N. Kim, Mr. Elanchezhiyan, J. Chae, Ys. Rhee, Mater. Sci. 28, 5014 (2010).

[2] K. Ramamoorthy, M. Arivanandhan, K. Sankaranarayanan, C. Sanjeeviraja, Mater. Chem. Phys. 85, 257 (2004).

[3] M.M. Ali, J. Basrah Res. 37, 49 (2011).

[4] S. Masuda, K. Kitamura, Y. Okumura, S. Miyatake, H. Tabata, T. Kawai, J. Appl. Phys. 93, 1624 (2003).

[5] A. Sholehah, A.H. Yuwono, C.R. Rimbani, Mater. Sci. Forum 737, 28 (2013).

[6] A. Kolodziejczak-Radzimska, T. Jesionowski, Materials (Basel) 7, 2833 (2014)

[7] S. Temel, F.O. Gokmen, E. Yaman, Eur. Sci. J. (ESJ) 13, 28 (2017).

[8] P.B. Taunk, R. Das, D.P. Bisen, R.K. Tamrakar, N. Rathor, Karbala Int. J. Mod. Sci. 1, 159 (2015).
[9] H. Khallaf, G. Chai, O. Lupan, H. Heinrich, S. Park, A. Schulte, L. Chow, J. Phys. D Appl. Phys. 42, 135304 (2009).

[10] M.J. Kim, C. Kim, S.H. Sohn, Mol. Cryst. Liq. Cryst. 677, 81 (2018).

[11] Y. Zhang, X.Y. Dang, J. Jin, T. Yu, B.Z. Li, Q. He, F.Y. Li, Y. Sun, Appl. Surf. Sci. 256, 6871 (2010).

[12] R. Bhowmik, M.N. Murty, E.S. Srinadhu, PMC Phys. $B$ 1, 20 (2008).

[13] Rahul, A.K. Verma, R.S.N. Tripathi, S.R. Vishwakarma, Natl. Acad. Sci. Lett. 35, 367 (2012).

[14] M.R. Khelladi, L. Mentar, M. Boubatra, A. Azizi, Mater. Lett. 67, 331 (2012).

[15] Z. Yuan, J. Electron. Mater. 44, 1187 (2015).

[16] K.L.P. Thi, L.T. Nguyen, N.H. Ke, D.A. Tuan, T.Q.A. Le, L.V.T. Hung, J. Electron. Mater. 47, 6302 (2018).

[17] G.S.A. Lghamdi, A.Z.A. Lzahrani, Middle East J. Sci. Res. 13, 1144 (2013)

[18] Z.R. Khan, M.S. Khan, M. Zulfequar, M. Shahid Khan, Mater. Sci. Appl. 02, 340 (2011).

[19] R. Kumar, H. Rani, Int. Lett. Chem. Phys. Astron. 14, 26 (2013)

[20] T. Hurma, J. Mol. Struct. 1189, 1 (2019). 